\title{
Critical reflections on methodological challenge in arts and dementia evaluation and research
}

Paper submitted to Dementia: the international journal of social research and practice 14/07/17. Accepted 01/09/17.

\section{Karen Gray}

Association for Dementia Studies, University of Worcester, UK

\section{Simon Chester Evans}

Association for Dementia Studies, University of Worcester, UK

\section{Amanda Griffiths}

Division of Psychiatry and Applied Psychology, University of Nottingham, UK

\section{Justine Schneider}

Institute of Mental Health, University of Nottingham, UK

\begin{abstract}
Methodological rigour, or its absence, is often a focus of concern for the emerging field of evaluation and research around arts and dementia. However, this paper suggests that critical attention should also be paid to the way in which individual perceptions, hidden assumptions and underlying social and political structures influence methodological work in the field. Such attention will be particularly important for addressing methodological challenges relating to contextual variability, ethics, value judgement, and signification identified through a literature review on this topic. Understanding how, where and when evaluators and researchers experience such challenges may help to identify fruitful approaches for future evaluation.
\end{abstract}

This paper is based upon a presentation on the subject given at the First International Research Conference on the Arts and Dementia: Theory, Methodology and Evidence on 9 March 2017.

\section{Keywords}

arts evaluation methodology methodological challenge epistemology methods complexity

\section{Corresponding author}

Karen Gray The Association for Dementia Studies, Institute of Health and Society, St Johns Campus, University of Worcester, Worcester WR2 6AJ, UK. Email: karen.gray@worc.ac.uk 


\section{Critical reflections on methodological challenge in arts and dementia evaluation and research}

Two commonplaces about the arts and dementia: (1) it works, and; (2) this is hard to 'prove'. Arts organisations, museums and heritage bodies, and individual practitioners are delivering an increasing amount of arts activity designed to enhance the health and wellbeing of people living with dementia. There is a corresponding growth in research intended to strengthen the evidence base for this work and a requirement to tackle methodological challenges facing evaluators and researchers.

Methodology might be defined as the work that goes into identifying one's research position and methods. It has also been termed 'the description, the explanation and the justification of methods' (Kaplan, 1964, p. 18). Kaplan's definition is highlighted because it draws attention to the subjective and dialogical nature of methodological work. Choices about methods are made in social, political and historical contexts. It has further been suggested that research methods are essentially performative, that 'they have effects; they make differences; they enact realities; and they can help to bring into being what they also discover' (Law \& Urry, 2004, p. 393). This paper reflects on some implications of acknowledging these aspects of methodological work through consideration of the challenges facing researchers and evaluators of arts-based activities for people with dementia. Key challenges were identified through a critical literature review for an ongoing project whose aim is to signpost solutions for evaluation research practice.

Despite promising and positive findings, many arts and dementia studies will be dismissed because of alleged lack of 'rigour' and poor methodological quality (Vink, Birks, Bruinsma, \& Scholten, 2011). Underpinning theoretical frameworks are said to be insufficient (Beard, 2011; Young, Camic, \& Tischler, 2016). Causal mechanisms are not well understood (Windle et al., 2014). Persuasive economic evaluation appears elusive, perhaps because of a lack of research designs suitable for robust comparative cost analysis and able to satisfactorily address questions of causality (Fujiwara, Kudrna, \& Dolan, 2014). Finally, evaluators and researchers have failed to access the direct experience of people with dementia (Zeilig, Killick, \& Fox, 2014). Overall the concern is that funders, commissioners and policymakers remain unconvinced of the value of investment (Fraser, Bungay, \& Munn-Giddings, 2014). 
In facing such issues, and disagreement about the best approach to future evaluation and research practice, arts and dementia is aligned with the wider field of arts and health research where, despite some ambivalence about it from those who deliver arts activities, the dominant discourse remains that of the health sciences (Daykin, Gray, McCree, \& Willis, 2017; Fancourt \& Joss, 2014). Greater methodological rigour is advocated by those who view the primary aim of research as investigating whether and how arts activity might be integrated within evidence-based practice. Robust experimental designs, including the randomised controlled trial (RCT), are seen as desirable (Moniz-Cook, Vernooij-Dassen, Woods, Orrell, \& INTERDEM Network, 2011). Mixed method studies combine the strengths or compensate for the limitations of both quantitative and qualitative methods and are popular in the wider field of public health (Pluye \& Hong, 2014). Standard qualitative methods are commonly used on their own in this field to explore nuanced implications of the effects of the arts. While it is sometimes thought that these methods will be less acceptable in health contexts, this may not reflect the views of all health commissioners (Goulding, 2014). Assessment of artistic quality, aesthetic experience, cultural contributions, process, social impact and economic value appear rare in the arts and dementia literature. Social or experiential effects of the arts on individuals with dementia have been explored using methodologies including ethnography (Swinnen, 2014), interpretive phenomenological analysis (Gregory, 2011), personal narrative analysis (Fels \& Astell, 2011) and participatory critical arts-based enquiry (Dupuis, Kontos, Mitchell, Jonas-Simpson, \& Gray, 2016). However, such distinctive approaches are seldom employed in evaluating the impact, outcomes or implementation of arts and dementia activities.

\section{The challenges of conducting a literature review about methodological challenge}

What methodological challenges are experienced by researchers and evaluators of arts-based activities delivered for and with people living with dementia? One difficulty with conducting research around this question is that challenges might not be visible except 'in the gaps'through reflection on what cannot be said (perhaps because it isn't considered publishable by a particular journal), or what has not been done in a study (maybe because it didn't get past the ethics review process, or was too expensive). A reading of the limitations in arts and dementia research studies might lead us to believe that proper application of method is particularly problematic for positivist researchers. It is useful to remember that clear signposting of research challenges may not always be presented by those approaching the subject from other perspectives, where issues such as researcher 'reflexivity' will be addressed, but not necessarily framed as limitations. 
The main inclusion criterion for the review was that literature should be a published, empirical study reporting on the impacts, outcomes or implementation of arts-based activity for people with dementia. The process of defining criteria became a microcosm for debates in the field in general. What is, and is not, 'art'? What is 'activity'? What constitutes 'dementia'? It is worth noting that language matters greatly, and not just in the context of a literature review. A word used in one field or discipline may have different implications in another. The lack of a clear distinction between - for example - what constitutes 'therapy' or 'activity' in both arts and research practice has been identified as contributing to charged debate in arts and health, even placing an 'unreasonable' burden of expectation on arts practice (Broderick, 2011).

Methodological terms, such as 'intervention', used without careful definition, may lead to miscalibration of judgements about study quality and the evidence produced (Petticrew, Viehbeck, Cummins, \& Lang, 2016).

Online database searches were conducted in February 2016. After application of exclusion criteria, hand-searching and consultation with others, 75 journal papers, referencing both quantitative and qualitative studies, were set aside for detailed reading in June 2016. Fourteen systematic or other reviews focusing on arts activity for people with dementia were added. A narrative approach for the review, informed by critical synthesis methods, was chosen as best suiting the topic (Dixon-Woods, 2006; Thomas \& Harden, 2007).

\section{Questions of methodological challenge}

Because methodology is seen as a process of 'justification, description and explanation', key patterns of challenge identified through the review are presented here as questions, drawing attention to their dialogical nature.

\section{How do we account for complex contexts?}

Context has been defined as 'anything external to the intervention that may act as a barrier or facilitator to its implementation, or its effect' (Moore et al., 2015a, p.2). Also in relation to implementation, Damschroder and colleagues (2009) apply the term to the broad set of interacting variables active in the circumstances surrounding an intervention, including its specific setting. Understanding the processes informing these interactions may help in assessment of fidelity and quality of implementation, clarification of causal mechanisms, and 
interpretation of the findings of evaluation (Moore et al., 2015). It has been suggested that attention to process more generally, particularly at the level of organisational barriers and opportunities for implementation of activities, would enable arts and health researchers to contribute more effectively to the development and sustainability of practice and research (Clift, 2012). As has been noted, this area appears under-developed in the emerging field of arts and dementia where projects are often studied at pilot or developmental stage. The reasons for this may be structural since, as Goulding (2014) has noted in relation to arts on prescription for older people, the delivery of arts projects is often shaped by the scarcity of long-term strategic funding and the prevalence of small-scale, local or opportunistic implementations.

Many types of contextual interactions of interest emerged from the review. These include: the setting where the arts activity is experienced, its physical space, routines and associated practices; time - including the timing of individual iterations of an activity and developmental stage of the intervention; the cultural, social and relational networks of participants; the personal and clinical histories, cultural demographies and aesthetic and other preferences of participants, and; the policies, practices and discourse surrounding dementia and dementia care. In the studies reviewed, these elements constantly interact with the artistic, technical and relational practices of individual arts practitioners and organisations.

Arts-based activities for people with dementia are often considered and evaluated as 'complex interventions'. Advocates of a realist approach have suggested that while the influential Medical Research Council (MRC) guidance on the evaluation of complex interventions (Craig et al., 2008) discusses ways in which evaluation may be used to build theory and understand causal mechanisms, it makes insufficient mention of the role of context for implementation (Fletcher et al., 2016). It has separately been argued that commonly applied definitions of 'complexity' may not fully engage with understandings of the concept from a systems perspective (Rickles, Hawe, $\&$ Shiell, 2007), and that such engagement could be useful in supporting effective implementation and evaluation (May, Johnson, \& Finch, 2016; Pawson, 2013). A re-framing of the concept of complexity in the light of these insights might see evaluators treating an arts for dementia activity as a critical event occurring within a complex adaptive system (Hawe et al. 2009), or, as a complex intervention inserted into a complex system (Pawson, 2013). Such treatments would lead to a view of contextual variations as elements integral to successful implementation rather than problems for the experimental method. 
The capture, documentation, analysis and reporting of descriptive detail about an arts activity is itself challenging. To this challenge, we could add that of dealing, methodologically, with uncertainty when attempting to understand causality within the complex systems in which arts for dementia activities are introduced.

\section{How do we address issues of value?}

Any choice of evaluation question is likely to be, as Weber suggests, rendered significant in light of 'the cultural values with which we approach reality' (Weber, 1949, p. 78). An evaluator of artsbased activities for people with dementia will make value judgements about the nature and quality of evidence required, arts and culture in general and the specific arts activity, and also about the condition of dementia, the people who experience it and dementia care practice. We may disagree about whether something has intrinsic value or whether the value is realised instrumentally through an action whose impact is felt by someone or something. Something may be ascribed a quantity of value, and we also engage in acts of valuing. An evaluator engages in one such act: judging how well some value, implicit in the theory informing a project, has been expressed through its implementation in practice. However, the reason why a particular set of values has been arrived at is rarely articulated in evaluation or research studies.

The differing focuses of the studies reviewed demonstrate that arts activities for people with dementia are variously valued because they:

- Provide an alternative to pharmacological and psychosocial interventions aimed at 'managing' the behavioural and psychological symptoms of dementia;

- Have specific and measurable cognitive, social or emotional benefits;

- Improve communication and interaction between carers and people with dementia or lessen the 'burden' of care;

- Enhance subjective wellbeing or quality of life of people living with dementia or their caregivers;

- Offer opportunities for personally enjoyable and meaningful activity;

- Challenge dominant narratives about dementia and constructions of the self in dementia;

- Enrich communities through cultural inclusion and opportunities for engaged citizenship. 
While it has been shown that the arts may be a cost effective way to improve quality of life for older people (Coulton, Clift, Skingley, \& Rodriguez, 2015), the reviewed literature contained no economic studies.

The diversity and multiplicity of values identified, sometimes within a single study, suggests that a correspondingly rich and varied set of methodological tools is required to investigate them, and to unpick the implications of unreported value judgements or assumptions.

\section{What makes for an ethical evaluation?}

The involvement of persons living with dementia in research, including those lacking capacity, is an evolving issue. In the UK, the Mental Capacity Act 2005 provides researchers with a framework and code of practice (Department for Constitutional Affairs, 2007). The wishes of people with dementia are rightly recognised, alongside a requirement to support their continued individual participation in society. It has long been suggested that there are ethical questions to be answered around the delivery of arts activities effecting a change where a participating subject has not given, or is not able to give consent (Matarasso, 1996). If it fails to address such issues, evaluation research could be viewed as an activity 'done to' participants with dementia, with or without their consent (Bryden, 2015).

Thinking around personhood encourages us to view all behaviour of people living with dementia as meaningful. People with dementia might not lack capacity to communicate their opinions and feelings (Mozley, Huxley, Sutcliffe, Bagley, \& Burns, 1999) but there is little indication in the published literature that they are routinely consulted about or included in the development, implementation, or evaluation of activities. Participatory and wider stakeholder co-produced research processes may be of value for arts and health (Daykin et al., 2017) but appear uncommon within arts and dementia.

Institutional ethics processes are, moreover, described as cumbersome, time-consuming (a particular issue for evaluation of existing projects) and potentially exclusionary (Murray, 2013; Nuffield Council on Bioethics, 2009). Their processes, largely designed around clinical research models, may not sit well with low risk research and evaluation in arts and dementia contexts. 


\section{How is meaning communicated and interpreted?}

Any exploration of our engagements in and with art will challenge us to think deeply about signification - the way in which meaning is conveyed and expressed. Art does not always give up its meaning easily, and the kind of meaning-making that takes place when we 'do art' happens at unexpected times and places: within the practice of the artist or in the production of an artwork, in a momentary interaction experienced by an individual engaging with art or with an arts practitioner or subsequently in the 'story' of that individual. Recognising, capturing and then making sense of significant moments so that they may be evaluated can be difficult, particularly when the person engaging with art has diminished cognitive ability, language skills and difficulties with attention (van Baalen, Vingerhoets, Sixma, \& de Lange, 2010). This point is illustrated in a project exploring art-viewing and making in a US art gallery in which a care partner reportedly told the researcher that a person with dementia had not understood what was going on during the art-viewing phase of an activity (Burnside et al., 2015). This might make us question what it means, methodologically, that someone engaging in an activity does not 'understand' that activity in the way that an evaluator (or an artist, or a care partner) does.

Researchers and evaluators report difficulties in using direct research methods with people with dementia (Cridland, Phillipson, Brennan-Horley, \& Swaffer, 2016), some of which may originate in societal stigma. The effect of stigma in relation to participation in research has been documented (Batsch \& Mittelman, 2012; Swaffer, 2014). One implication is that research activity might need to explore models of enquiry that embrace and empower communication and capability rather than cognitive failure or impairment.

\section{Conclusions}

Rigorous application of method is only one problematic area for research into arts-based activities for people with dementia. Challenges for documentation and analysis of the complex contexts in which activities are delivered also require consideration, not least because of how addressing them could inform the shape of future projects and our understanding of how change is effected. There may be an ethical imperative to find modes of enquiry which empower the communication capabilities of people living with dementia, and to explore ethical processes that more fully allow their informed involvement. In addition, evaluators and researchers may benefit from considering the impact for methodology of assumptions about value. 
Stakeholder dialogue around the use of flexible methodological approaches may help us to understand better what happens when people with dementia engage with art, and to ensure that art is used effectively. Attempts to address the identified challenges could lead, for example, to the use of ethnographic, participatory or narrative approaches alongside implementation and process or realist evaluation methodologies. Questioning the assumed usefulness of hierarchies of evidence, Petticrew and Roberts remind us that "[e]nd point users, policy makers, and practitioners in particular ask many questions about interventions that are not just about effectiveness" (Petticrew \& Roberts, 2003, p. 523). So, what other questions might we need to be asking, and who might we need to be listening to, in our drive to move on from the it works, but it's hard to prove it' commonplaces?

These reflections on methodological challenge in arts and dementia suggest that further research could usefully explore how and why methodological choices are made, what pragmatic, political or personal factors determine these choices and the success of the resulting work, how other stakeholders are involved, if and how the cross-disciplinary nature of the arts and dementia field is reflected in the process, whether tensions result, and how these might be resolved. This may be particularly useful if, with Law and Urry (2004), we suspect that methods not only uncover realities, but also produce them.

\section{Acknowledgements}

The authors would like to thank those who offered responses and discussion at the RSPH First International Research Conference on the Arts and Dementia following the presentation upon which this paper is based. This work is supported by the Alzheimer's Society, through funding of a doctoral studentship.

\section{Conflict of interest}

The authors declare that there is no conflict of interest.

\section{References}

Batsch, N. L., \& Mittelman, M. S. (2012). World Alzheimer Report 2012: Overcoming the stigma of dementia. Alzheimer's Disease International. London. 
Beard, R. (2011). Art therapies and dementia care: A systematic review. Dementia: The International Journal of Social Research and Practice, 11(5), 633-656.

Broderick, S. (2011). Arts practices in unreasonable doubt? Reflections on understandings of arts practices in healthcare contexts. Arts \& Health, 3(2), 95-109.

Bryden, C. (2015). Nothing about us without us! 20 years of dementia advocacy. London: Jessica Kingsley Publishers.

Clift, S. (2012). Creative arts as a public health resource: moving from practice-based research to evidence-based practice. Perspectives in Public Health, 132(3), 120-127.

Coulton, S., Clift, S., Skingley, A., \& Rodriguez, J. (2015). Effectiveness and cost-effectiveness of community singing on mental health-related quality of life of older people: Randomised controlled trial. British Journal of Psychiatry, 207(3), 250-255.

Craig, P., Dieppe, P., Macintyre, S., Michie, S., Nazareth, I., \& Petticrew, M. (2008). Developing and evaluating complex interventions: the new Medical Research Council guidance. BMJ, 337, a1655.

Cridland, E. K., Phillipson, L., Brennan-Horley, C., \& Swaffer, K. (2016). Reflections and recommendations for conducting in-depth interviews with people with dementia. Qualitative Health Research, 26(13), 1774-1786.

Damschroder, L. J., Aron, D. C., Keith, R. E., Kirsh, S. R., Alexander, J. A., \& Lowery, J. C. (2009). Fostering implementation of health services research findings into practice: a consolidated framework for advancing implementation science. Implementation Science, 4(1), 50.

Daykin, N., Gray, K., McCree, M., \& Willis, J. (2017). Creative and credible evaluation for arts, health and well-being: opportunities and challenges of co-production. Arts \& Health: An International Journal for Research, Policy and Practice, 9(2), 123-138.

Department for Constitutional Affairs. (2007). Mental Capacity Act 2005 Code of Practice. London.

Dixon-Woods, M. (2006). How can systematic reviews incorporate qualitative research? A critical perspective. Qualitative Research, 6(1), 27-44.

Dupuis, S. L., Kontos, P., Mitchell, G., Jonas-Simpson, C., \& Gray, J. (2016). Re-claiming citizenship through the arts. Dementia: The International Journal of Social Research and Practice, 15(3), 358-380.

Fancourt, D., \& Joss, T. (2014). Aesop: A framework for developing and researching arts in health programmes. Arts \& Health: An International Journal for Research, Policy and Practice, 7(1), 
$1-13$.

Fels, D. I., \& Astell, A. J. (2011). Storytelling as a model of conversation for people with dementia and caregivers. American Journal of Alsheimer's Disease \& Other Dementias, 26(7), $535-541$.

Fletcher, A., Jamal, F., Moore, G., Evans, R. E., Murphy, S., \& Bonell, C. (2016). Realist complex intervention science: Applying realist principles across all phases of the Medical Research Council framework for developing and evaluating complex interventions. Evaluation, 22(3), 286-303.

Fraser, A., Bungay, H., \& Munn-Giddings, C. (2014). The value of the use of participatory arts activities in residential care settings to enhance the well-being and quality of life of older people: A rapid review of the literature. Arts \& Health: An International Journal for Research, Policy and Practice, 6(3), 266-278.

Fujiwara, D., Kudrna, L., \& Dolan, P. (2014). Quantifying and valuing the wellbeing impacts of culture and sport. Department for Culture, Media \& Sport Research Paper. London.

Goulding, A. (2014). Arts on prescription for older people: Different stakeholder perspectives on the challenges of providing evidence of impact on health outcomes. Journal of Applied Arts \& Health, 5(1), 83-107.

Gregory, H. (2011). Using poetry to improve the quality of life and care for people with dementia: A qualitative analysis of the Try to Remember programme. Arts \& Health: An International Journal for Research, Policy and Practice, 3(2), 160-172.

Kaplan, A. (1964). The conduct of inquiry; methodology for behavioral science. San Francisco: Chandler Publishing Company.

Law, J., \& Urry, J. (2004). Enacting the social. Economy and Society, 33(3), 390-410.

Matarasso, F. (1996). Defining values: Evaluating arts programmes. The Social Impact of the Arts. Stroud, Comedia.

May, C. R., Johnson, M., \& Finch, T. (2016). Implementation, context and complexity. Implementation Science, 11(1), 141.

Moniz-Cook, E., Vernooij-Dassen, M., Woods, B., Orrell, M., \& INTERDEM Network. (2011). Psychosocial interventions in dementia care research: The INTERDEM manifesto. Aging \& Mental Health, 15(3), 283-290.

Moore, G. F., Audrey, S., Barker, M., Bond, L., Bonell, C., Hardeman, W., ... Baird, J. (2015). Process evaluation of complex interventions: Medical Research Council guidance. BMJ, 
350, h1258.

Mozley, C., Huxley, P., Sutcliffe, C., Bagley, H., \& Burns, A. (1999). 'Not knowing where I am doesn't mean I don't know what I like': Cognitive impairment and quality of life. International Journal Of Geriatric Psychiatry, 14, 776-783.

Murray, A. (2013). The Mental Capacity Act and dementia research. Nursing Older People, 25(3), $14-20$.

Nuffield Council on Bioethics. (2009). Dementia: Ethical issues. London.

Pawson, R. (2013). The science of evaluation: A realist manifesto. London: Sage.

Petticrew, M., \& Roberts, H. (2003). Evidence, hierarchies, and typologies: Horses for courses. Journal of Epidemiology and Community Health, 57(7), 527-529.

Petticrew, M., Viehbeck, S., Cummins, S., \& Lang, T. (2016). Å mêmes mots, sens différents - les difficultés de la terminologie épidémiologque avec la recherche en interventions en santé des populations. Revue d'Épidémiologie et de Santé Publique, published online 31 March 2016.

Pluye, P., \& Hong, Q. N. (2014). Combining the power of stories and the power of numbers: Mixed methods research and mixed studies reviews. Annual Review of Public Health, 35(1), $29-45$.

Rickles, D., Hawe, P., \& Shiell, A. (2007). A simple guide to chaos and complexity. Journal of Epidemiology \& Community Health, 61(11), 933-937.

Swaffer, K. (2014). Dementia: Stigma, language, and dementia-friendly. Dementia: The International Journal of Social Research and Practice, 13(6), 709-716.

Swinnen, A. M. C. (2014). Healing words: A study of poetry interventions in dementia care. Dementia: The International Journal of Social Research and Practice, 15(6), 1377-1404.

Thomas, J., \& Harden, A. (2007). Methods for the thematic synthesis of qualitative research in systematic reviews (NCRM Working Paper Series No. 10). London.

van Baalen, A., Vingerhoets, A. J. J. M., Sixma, H. J., \& de Lange, J. (2010). How to evaluate quality of care from the perspective of people with dementia: An overview of the literature. Dementia: The International Journal of Social Research and Practice, 10(1), 112-137.

Vink, A. C., Birks, J. S., Bruinsma, M. S., \& Scholten, R. J. (2011). Music therapy for people with dementia (Review). Cochrane Database of Systematic Reviews, (3), CD003477.

Weber, M. (1949). The methodology of the social sciences. (E. Shils \& H. Finch, Eds.). Glencoe, Illinois: The Free Press. 
Windle, G., Gregory, S., Newman, A., Goulding, A., O’Brien, D., \& Parkinson, C. (2014).

Understanding the impact of visual arts interventions for people living with dementia: A realist review protocol. Systematic Reviews, 3(91).

Young, R., Camic, P. M., \& Tischler, V. (2016). The impact of community-based arts and health interventions on cognition in people with dementia: a systematic literature review. Aging \& Mental Health, 20(4), 337-351.

Zeilig, H., Killick, J., \& Fox, C. (2014). The participative arts for people living with a dementia: a critical review. International Journal of Ageing and Later Life, 9(1), 7-34. 